\title{
Measurement of the electric fluctuation spectrum of magnetohydrodynamic turbulence
}

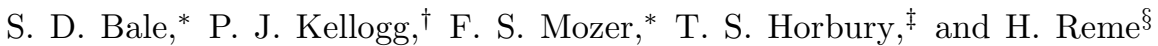

(Dated: October 26, 2018)

\begin{abstract}
Magnetohydrodynamic (MHD) turbulence in the solar wind is observed to show the spectral behavior of classical Kolmogorov fluid turbulence over an inertial subrange and departures from this at short wavelengths, where energy should be dissipated. Here we present the first measurements of the electric field fluctuation spectrum over the inertial and dissipative wavenumber ranges in a $\beta \gtrsim 1$ plasma. The $k^{-5 / 3}$ inertial subrange is observed and agrees strikingly with the magnetic fluctuation spectrum; the wave phase speed in this regime is shown to be consistent with the Alfvén speed. At smaller wavelengths $k \rho_{i} \geq 1$ the electric spectrum is softer and is consistent with the expected dispersion relation of short-wavelength kinetic Alfvén waves. Kinetic Alfvén waves damp on the solar wind ions and electrons and may act to isotropize them. This effect may explain the fluid-like nature of the solar wind.
\end{abstract}

PACS numbers: 52.35.Ra Plasma turbulence; 52.35.Bj Magnetohydrodynamic waves

Turbulence is ubiquitous in astrophysical plasmas; turbulent processes are thought to play a role in cosmic ray and energetic particle acceleration and scattering 1], advection dominated accretion flows, and perhaps solar/stellar wind acceleration. Yet key aspects of the physics of turbulence in magnetized plasmas are poorly understood, in particular the physics of dissipation at small scales. The classical scenario of magnetohydrodynamic turbulence is thus: fluctuations in the plasma are driven at some large 'outer' scale and decay by interacting locally in $k$-space. Eddies at some scale $k$ exchange energy with eddies at nearby spatial scales, possibly as a three-wave or higher order interaction 10, 11, 12], with the resulting net flow of energy to smaller spatial scales (larger $k$ ); this cascade of energy occurs over an 'inertial subrange' of $k$-space and can be shown to predict a power spectrum that scales as $k^{-5 / 3}$. At the smaller scale of the ion thermal gyroradius, $k \rho_{i} \geq 1$, the ions become demagnetized and the plasma can no longer behave as a simple fluid; the turbulent energy is then thought to be damped on the thermal plasma by Landau or transit-time damping. However, the details of this damping process are not known and there a few reported measurements in this regime of $k$-space.

Observations of the magnetic spectrum show breakpoints at near $k \rho_{i} \approx 1$, above which the spectrum typically becomes harder [13, 14, 15]. This has been interpreted variously as evidence of kinetic Alfvén waves [13], whistler wave dispersion 17], and ion cyclotron damping of Alfvén waves [16].

Here we report the first measured power spectrum of electric fluctuations in solar wind turbulence. The iner-

\footnotetext{
* Department of Physics and Space Sciences Laboratory, University of California, Berkeley

${ }^{\dagger}$ School of Physics and Astronomy, University of Minnesota, Minneapolis

¥The Blackett Laboratory, Imperial College, London, UK

$\S$ CESR, Toulouse, France
}

tial subrange is clearly evident and follows the magnetic fluctuation spectrum. At large wavenumbers $k \rho_{i} \geq 1$, the electric spectrum is enhanced.

Data are used from experiments on the Cluster spacecraft. Cluster flies four spacecraft, as a controlled tetrahedron, in an inclined orbit with apogee at 19 Earth radii $\left(R_{E}\right)$. From December to May each year, the spacecraft exit the terrestrial magnetosphere on the dayside and make measurements in the solar wind. We use approximately 195 minutes of data during the interval 00:07:00 - 03:21:51 on February 19, 2002, when Cluster was at apogee and spent several hours in the ambient solar wind; all of our data is from Cluster spacecraft 4 .

The electric field is measured by the Electric Field and Waves experiment (EFW) experiment [a]; EFW is a double-probe experiment which measures the floating voltage of $8 \mathrm{~cm}$ diameter current-biased spheres extended on $44 \mathrm{~m}$ wires in quadrature. These spheres, as well as the spacecraft, are illuminated by the Sun and emit photoelectrons which cause the surfaces to charge positive with respect to the plasma. The surfaces attract a return current of thermal electrons which provide the electrical coupling to the plasma. Systematic variations in this coupling, due to changing illumination or variations in surface properties and work function, are a large source of background noise in EFW at the spacecraft spin-period (4 seconds) and harmonics. This is discussed more below. EFW measures the electric field on two orthogonal sensor pairs in the spacecraft spin-plane at 25 samples/sec. These two components are rotated into $X$ and $Y$ components in the GSE (geocentric solar ecliptic) coordinate system. Since the GSE $Y$ direction represents the orientation with best symmetry for solar illumination, this component of the electric field is generally less noisy; we use the GSE $Y$ electric field $E_{y}$ for all of our analysis. However, at any given instance, $E_{y}$ is composed of data from all four electric field probes, each with slightly different photocoupling to the plasma. We therefore apply a finite impulse response (FIR) filter to the data to notch out the primary perturbations at the spin-tone and some 
harmonics.

The magnetic field is measured by the FGM instrument [2]; three-component magnetic field vectors are sampled at 22 samples/sec (SPS). In our analysis, we use the GSE $Z$ component of the magnetic field $B_{z}$ for reasons that are explained below. Moments of the solar wind ion distribution (velocity, density, and temperature) are computed from the ion spectrum measured by the CIS experiment [4].

Figure 1 shows an overview of the data used in the following analysis; panels a) and b) are wavelet spectrograms and will be discussed below. Panel c) shows the two components of measured electric field $E_{x}$ and $E_{y}$ in GSE coordinates. Panel (d) show the magnetic field data. Panels e), f), and g) show the plasma ion density, plasma ion $\beta_{i}$ (ratio of plasma to magnetic pressure), and Alfvén Mach number. The average ion beta is $\bar{\beta}_{i} \approx 5$, average Alfvén speed $\bar{v}_{A} \approx 40 \mathrm{~km} / \mathrm{s}$, and the average solar wind velocity is $\bar{v}_{s w} \approx(-347,4.9,-32.6) \mathrm{km} / \mathrm{s}$ (in GSE coordinates), over the entire interval. During the interval between 00:30 and 00:50, the magnetic field is nearly tangent to the Earth's bow shock (as per a calculation assuming straight field lines [3]); however, Cluster summary plots of electron and plasma wave data show no evidence of connection to the shock. All of our data is ambient solar wind.

To compute power spectra, the electric field data $E_{y}$ (25 samples/sec) were subsampled onto the time tags of the magnetic field data $B_{z}(22$ samples/sec) by linear interpolation; a total of exactly $2^{18}$ points are used. The power spectral density (PSD) was computed using both Fast Fourier Transform (FFT) and Morelet wavelet [9] schemes. The FFT was computed as follows: the data interval was divided into 64 contiguous ensembles of length 4096 (182 seconds); this gives an inherent bandwidth of $\Delta f \approx 1 / 186 \mathrm{~Hz}$. To minimize spectral leakage, each ensemble was 'whitened' by applying a first-order difference algorithm, the PSD was computed by FFT, then the spectrum was postdarkened [1] and divided by the bandwidth of the FFT. Since the data is prewhitened, no window function was applied before the FFT. The electric field spectra were then 'cleaned' by interpolating over the narrowband spikes resulting from the spin-associated signals described above. A final spectrum was computed as the average of the 64 ensembles. Figure 2 shows the FFT power spectra of $E_{y}$ and $B_{z}$ (in black). Wavelet spectra were computed by first producing the (complex) FFT of $E_{y}$ and applying the spectral cleaning (interpolation) to the real and imaginary parts, at positive and negative frequencies. An inverse FFT restores the 'cleaned' signal and a Morelet wavelet spectrogram was computed from this cleaned $E_{y}$, as well as the original $B_{z}$. The wavelet has $136 \log$-spaced frequencies; the final wavelet PSD is computed as the square of the spectrum averaged over time. The wavelet PSD is also shown in Figure 2 (in red). The wavelet spectrum extends to lower frequencies than the FFT, which is composed of ensembles of smaller data intervals; however, these very low frequencies lie be- low the 'cone of influence' and are unreliable $[9]$. Here we restrict our interpretation to the region where the FFT and wavelet spectra agree. The FFT electric spectrum in Figure 2 shows clearly the effect of the notch filters and residual spin-harmonic spikes. The wavelet PSD, with its much larger bandwidth, mostly averages over these residual features although a depression near the notched portion of the spectrum can be seen. The FFT and wavelet PSD spectra agree remarkably well for both electric and magnetic fields.

Of course, our (human) scheme of measuring time means little to the solar wind plasma, so there is little reason to expect the data to be inherently organized by a power spectrum in Hertz. Since the solar wind is superAlfvénic (Figure 1), the phase speed $v_{A}$ of the Alfvénic fluctuations is much less than the wind speed itself; hence the measured frequency spectrum is actually a Dopplershifted wavenumber spectrum $\omega \approx k v_{s w}$. This is often called Taylor's hypothesis and might not be considered to hold at large wavenumbers, especially if waves are present with phase speeds greater than the solar wind speed (such as whistler waves).

As discussed above, it is considered that the fluid-like behavior of the wind breaks down at near $k \rho_{i} \approx 1$, therefore $k \rho_{i}$ is a natural parameter for organization of the power spectrum. The top panel of Figure 3 shows the FFT and wavelet power spectra organized by $k \rho_{i}$, instead of frequency. For the FFT spectrum, the local values of $\left|v_{s w}\right|, T_{i}$, and $|B|$ are used to compute $k=\omega / v_{s w}$ and the thermal ion gyroradius $\rho_{i}=v_{i} / \Omega_{c i}$ averaged over each $(186 \mathrm{sec})$ ensemble; the $E_{y}$ and $B_{z}$ power spectra are then interpolated onto a linearly-spaced set of values $k \rho_{i} \in(0.006,10)$. Since solar wind parameters vary slightly in each ensemble, this also has the effect of smearing (averaging) over the narrowband interference in the FFT PSD of $E_{y}$. The wavelet spectrograms are timeaveraged on to 4 second intervals and then interpolated onto a set of log-spaced values of $k \rho_{i}$; panels a) and b) of Figure 1 show these scaled spectrograms as a function of time. In Figure $1 \mathrm{a}$ ) and b), the fluctuation power has been divided by $k^{-5 / 3}$ to highlight fluctuations above the average spectrum of the inertial range. The electric and magnetic wavelet spectrograms are then averaged to compute the composite spectra in panel a) of Figure 3.

Between $k \rho_{i} \approx 0.015$ and 0.45 , the wavelet and FFT spectra of electric and magnetic fluctuations show power law behavior with indices of $k^{-1.7}$, which is consistent with the Kolmogorov value of $5 / 3$. Both $\delta E_{y}$ and $\delta B_{z}$ show breakpoints at near $k \rho_{i} \approx 0.45$; the magnetic spectrum becomes harder with a index $k^{-2.12}$, while the electric spectrum becomes softer. As discussed above, hard magnetic spectra have been observed previously [14, 15]. Above $k \rho_{i} \approx 0.45$, the electric spectrum is power law like $k^{-1.26}$ to $k \rho_{i} \approx 2.5$. Above this second breakpoint, a exponential $\exp \left(-k \rho_{i} / 12.5\right)$ better fits the spectrum. At these higher wavenumbers, the electric field data is noisy and shows harmonics of the spin tone (as shown above). To test the validity of this data, we perform two analyses. 
The black dots of panel c) in Figure 3 show the correlation between the electric and magnetic wavelet power as a function of $k \rho_{i}$. It can be seen that the fluctuations are strongly correlated through the inertial range (with coefficient $\approx 1$ ), remain well-correlated between the two breakpoints $k \rho_{i} \in(0.45,2)$ and begin to loose correlation quickly above the second breakpoint. A wavelet cross-spectral analysis (between $\delta E_{y}$ and $\delta B_{z}$ was also computed; the blue bars show the cross-spectral coherence, with 1 sigma error bars, also as a function of $k \rho_{i}$. Again, $\delta E_{y}$ and $\delta B_{z}$ are strongly coherent through the inertial range and past the first breakpoint. We conclude that the electric and magnetic spectra physical and wellcorrelated up to the second spectral breakpoint. Above $k \rho_{i} \approx 2.5$ it is difficult to assess the quality of the data. If electrostatic waves are present, there is no expectation of correlation with $\delta B$; however in this initial study, we cannot eliminate the possibility of systematic noise at these frequencies. Additionally, the effects of low pass filters on both the EFW and FGM experiments may modify the spectra at these highest $\left(k \rho_{i}>3\right)$ frequencies.

To estimate the phase speed of the fluctuations, we use Faraday's law and compute the ratio of the electric and magnetic spectra. Since the electric field measurements are made in the spacecraft (unprimed) frame, we need to Lorentz transform to the plasma (primed) frame by $\vec{E}=\vec{E}^{\prime}+\vec{v}_{s w} \times \vec{B}$. Panel b) of Figure 3 shows the phase speed

$$
v_{\phi}\left(k \rho_{i}\right)=\frac{\delta E_{y}^{\prime}\left(k \rho_{i}\right)}{\delta B_{z}\left(k \rho_{i}\right)}=\frac{\delta E_{y}\left(k \rho_{i}\right)}{\delta B_{z}\left(k \rho_{i}\right)}+\bar{v}_{x}-\bar{v}_{z} \frac{\bar{B}_{x}}{\bar{B}_{z}}
$$

where $\bar{v}_{x}, \bar{v}_{z}, \bar{B}_{x}$, and $\bar{B}_{z}$ are the average $x$ and $z$ components of the solar wind velocity and magnetic field. The black dots in panel b) are computed from the wavelet spectrum, while the blue line is computed from the FFT spectrum. The average Alfvén speed $\bar{v}_{A} \approx 40 \mathrm{~km} / \mathrm{s}$ is shown as a horizontal bar. Over, and even below, the inertial range $k \rho_{i} \in(0.015,0.4)$ the phase speed is consistent with the local Alfvén speed; this is strong evidence of the Alfvénic nature of the cascade. The red curve in panel b) is a fit of the function $v_{0}\left(1+k^{2} \rho_{i}^{2}\right)$ to the FFT curve, where $v_{0}$ is a free parameter which finds a best fit at $v_{0} \approx 55 \mathrm{~km} / \mathrm{s}$. This function approximates the dispersion relation of kinetic Alfvén waves. The cold-plasma whistler wave phase speed goes as $v_{\phi} \approx\left(k \rho_{i}\right) \beta^{-1 / 2} v_{A}$ above $\omega>\Omega_{c i}$, i.e. linear with $k \rho_{i}$, and would form a much shallower dispersion above $k \rho_{i} \approx 1$ than is observed in panel b) of Figure 3. This leads us to believe that the Alfvén waves in the inertial subrange eventually disperse as 'kinetic' Alfvén waves above $k \rho_{i} \approx 1$, becoming more electrostatic and eventually damping on the thermal plasma. Plasma heating by linear dissipation of kinetic Alfvén waves at $\beta \approx 1$ has been studied in the context of accretion flows $[\underline{5}]$. There it was found that Landau and transit-time damping contribute to both proton and electron heating at short wavelengths, which is enhanced for higher $\beta$. Kellogg [7] computed the level of electric field fluctuations required to stochastically thermalize protons to $1 \mathrm{AU}$ in the solar wind; he found that a spectral density of $E^{2} \approx 10^{-11}(\mathrm{~V} / \mathrm{m})^{2} \mathrm{~Hz}^{-1}$ was sufficient. This is one order of magnitude less than our observed levels (Figure 2). It is, therefore, plausible to conclude that the observed electric spectrum is responsible for isotropizing the solar wind protons and may be the mechanism by which the solar wind maintains its fluid-like characteristics.

\section{Acknowledgments}

Cluster data analysis at UC Berkeley is supported by NASA grants NAG5-11944 and NNG04GG08G.
[1] Bieber et al., J. Geophys. Res., 98, 3585, 1993.

[2] A. Balogh et al., Space Science Rev., 79, 65, 1997.

[3] Filbert, P. C. and P. J. Kellogg, J. Geophys. Res., 84, 1369, 1979.

[4] H. Reme et al., Space Science Rev., 79, 303, 1997.

[5] G. Gustafsson et al., Space Sci. Rev., 79, 137 (1997).

[6] G. Gustafsson et al., Space Sci. Rev., 79, 137 (1997).

[7] P. J. Kellogg, Astrophys. J., 528, 480, 2000.

[8] E. Quataert, Astrophys. J., 500, 978, 2000.

[9] C. Torrence and G. P. Compo, Bull. American Meteorological Society, 79, 61, 1998.

[10] F. Waleffe, Phys. Fluids, 4, 350, 1992.

[11] P. Goldreich and S. Sridhar, Astrophys. J., 485, 680, 1997.

[12] C. S. Ng and A. Bhattacharjee, Astrophys. J., 465, 845, 1996.

[13] R. J. Leamon et al., J. Geophys. Res., 104, 22331, 1999.

[14] R. J. Leamon et al., J. Geophys. Res., 103, 4475, 1998.
[15] H. J. Beinroth and F. M. Neubauer, J. Geophys. Res, 86, 7755, 1981.

[16] S. P. Gary, J. Geophys. Res., 104, 6759, 1999.

[17] O. Stawicki, S. P. Gary, and H. Li, J. Geophys. Res., 106, 8273, 2001. 
FIG. 1: Wavelet and time series data of solar wind turbulence. From the top-down, the five panels show a) the wavelet spectrogram of $E_{y}$, as a function of $k \rho_{i}$, b) a similar wavelet spectrogram of $\left.B_{z}, \mathrm{c}\right)$ the $X$ and $Y$ components of the measured electric field, d) the vector magnetic field, e) plasma ion density, $\mathrm{f}$ ) plasma ion $\beta$, and $\mathrm{g}$ ) the Alfvén Mach number. This entire interval was used for the spectral analysis of $E_{y}$ and $B_{z}$. The spectral breakpoints called out.

FIG. 2: Power spectral density of electric $\delta E_{y}$ and magnetic fluctuations $\delta B_{z}$ as a function of frequency, computed from FFT (black) and Morlet wavelet (red) algorithms. The FFT spectrum of electric field (upper panel) shows the effect of notch filters and residual spin-tone data.
FIG. 3: The wavelet (upper) and FFT (lower) power spectra of $E_{y}$ (green) and $B_{z}$ (black) binned as a function of wavenumber $k \rho_{i}$ (and offset for clarity) in panel a). The electric are multiplied by factor to lie atop the magnetic spectra. The spectrum is Kolmogorov $k^{-5 / 3}$ over the interval $k \rho_{i} \in(0.015,0.45)$; a spectral breakpoint occurs for both $E_{y}$ and $B_{z}$ at $k \rho_{i} \approx 0.45$. A second breakpoint occurs for electric spectrum at $k \rho_{i} \approx 2.5$ above which the electric spectrum is more exponential. Panel b) shows the ratio of the electric to magnetic spectra in the plasma frame; the average Alfvén speed $\left(\bar{v}_{A} \approx 40 \mathrm{~km} / \mathrm{s}\right)$ is shown as a horizontal line. The red line is a fitted dispersion curve, discussed in the text. Panel c) shows both the cross-coherence of $\delta E_{y}$ with $\delta B_{z}$ (as blue dots with error bars) and the correlation between the electric and magnetic power (as black dots). 
This figure "figure1.png" is available in "png" format from: http://arxiv.org/ps/physics/0503103v1 
This figure "figure2.png" is available in "png" format from: http://arxiv.org/ps/physics/0503103v1 
This figure "figure3.png" is available in "png" format from: http://arxiv.org/ps/physics/0503103v1 